UCRL- 92160

PREPRINT

\title{
AN IMPROVED APPROACH TO CHARACTERIZING AND \\ PRESENTING STREAK CAMERA PERFORMANCE
}

\section{J. D. Wiedwald}

\author{
B. A. Jones
}

This paper was prepared for submittal to

SPIE's 29 th Annual Internationa? Technical Symposium, San Diego, CA

August 18-23, 1985

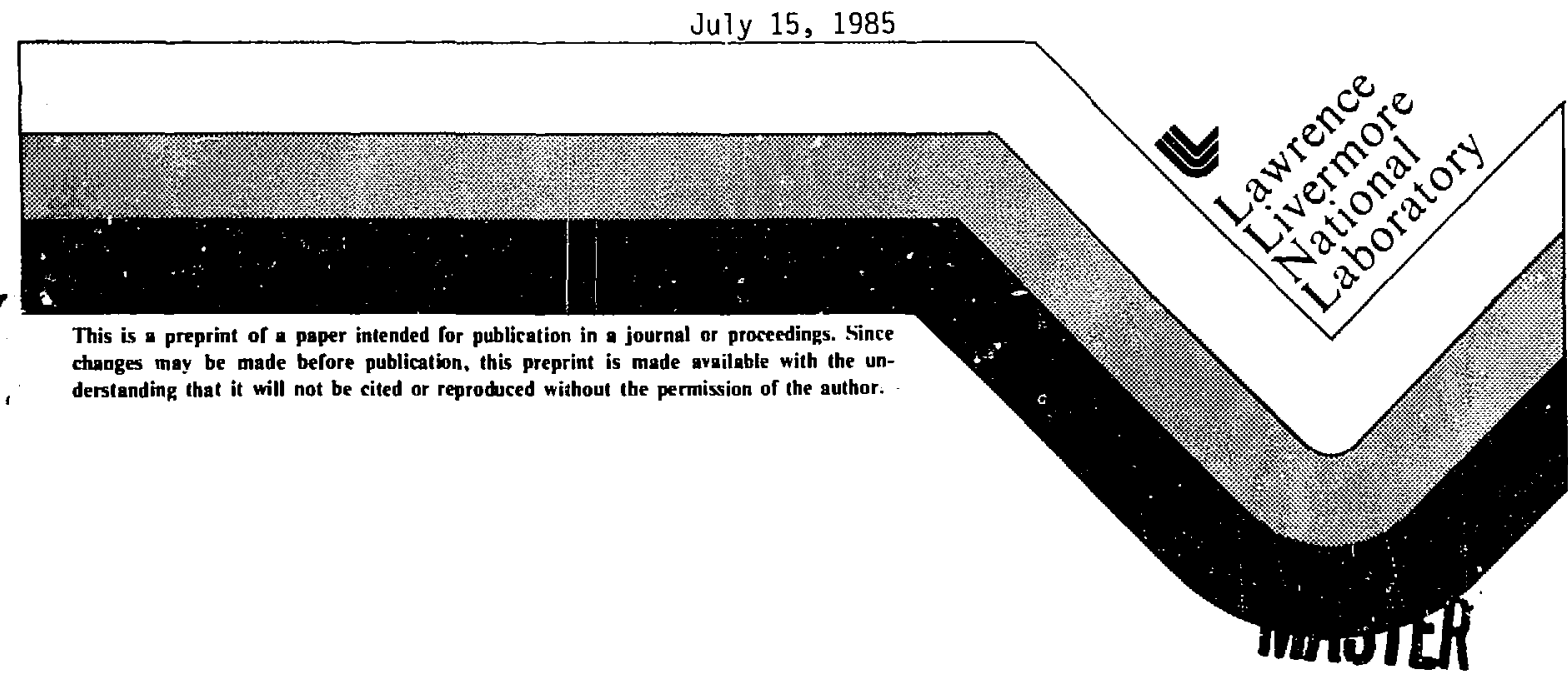

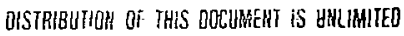

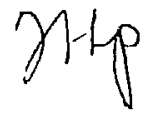


An improved approach to charactevizing and presenting streak camera performance

J. D. Wiedwald and B. A. Jones

Lawrence Livermore National Laboratory

Laser Fusion Program

P. 0 . Box $5508, L-479$

Livemore, California 94550

(415) $422-1338$
UCRL- -92160

DE85 015188

\section{Abstract}

Tne performance of a streak. camera recording system is strongly lirixed to the technicue used to amplify, detect and quantify the streaked image. At the Lawrence Livermore National Laboratory (LLNL) streak camera images have been recorded both on $\mathrm{film}$ and by fiber-optically coupling to charge-coupled devices (CCD's). During the development of a new process for recording these images (lens coupling the image onto a cooled CCD) the definitions of important perfornance characteristics such as resolution and dynamic range were re-examined. As a result of this development, these performance characteristics are now presented to the streak camera user in a more useful format than in the past. This paper describes how these techniques are used within the Laser fusion Program at LLNL. The system resoivtion is presented as a modulation trarsfer function, including the seldom reported effects that flare and light scattering have at low spatial frequencies. Data are presented such that a user can adjust image intensifier gain and pixel averaging to optimize the useful dynamic range in any particular application.

\section{The LLNL streak camera system}

The Laser Fusion Program at the Lawrence Livermore National Laboratory uses an internally designed streak camera as 3 principal diagnosicic instrument. This streak cameral utilizes the RCA C 73435 image converter tube in several configurations as its streaking element. Various photocathodes are employed to detect photons having energies ranging from $1.1 \mathrm{eV}(1060 \mathrm{~nm})$ to $>10 \mathrm{keV}$. To amolify the image to a recordable intensity an ITT F4113 intensifier tube with an MCP (micro-channel plate) and fiber optic input and output windows is coupled to the output of the image converter tube. Until recently, the image was recorded either directly onto film or fiber-optically coupled to a two-dimensional charge-coupled device.

We have recently developed a streak camera readout system based on a themo-electrically cooled CCD camera ${ }^{2}$ manufactured by Photometrics, Ltd. (Tucson, $A Z$ ). The output of the image intensifier is lens coupled to the CCD camera by an fl.2 lens. Lens coupling was chosen over fiber optics to minimize mechanical and thermal stress on the CCD. The linearity and large dynamic range of the CCD camera, coupled with rapid data analysis capability, allow us to characterize the streak camera system more thoroughly than in the past.

\section{How streak cameras are used in the Laser Fusion Program}

Sticeak cameras are used to record the temporal history of a radiation flux related to either the laser target heating heam or to the effects of that beam on a target. in addition to the sweep direction, the orthogonal axis is often used to display spatial or chromatic information by using a microscope or spectrometer in front of the streak camera. In some cases there are multiple signal channels across the streak camera slit, provided by optical fibers carrying independent signals. These applications call for high spatial resolution while temporal resolution requirements are typically not severe, 25-50 psec is usually adequate. As a result, streak camera sweep speeds are usually in the 1.5 to $10 \mathrm{nsec}$ range.

Laser fusion experiments occur relatively infrequently, once or twice a day is typical, and represent a large investment per shot. Due to uncertainty in target performance, signal levels are soinewhat unpredictable. Therefore a large and well-defined measuring system dynamic range is particularly beneficia1. The experimenter wants to know where the limits of the dynamic range are, what happens if those limits are exceeded and how to best adjust the system gain, both preceding and following the streak camera. In many cases the experimenter also wants to know how system spatial resolution will affect the diagnostic measurement, which will result in channel crosstalk, spectral line broadening or degradation of spatial resolution of an object source.

By using the new CCO camera we now generate a family of system transfer functions that describe the noise and non-linearity characteristics of the streak camera system at various image intensifier gains. We also measure the modulation transfer function (MTF) in the spatial direction at the streak camera input and both spatial and temporal axes at the output of the image intensifier. With this information, the user can determine the accuracy and limitations of the streak camera system as it is being applied. 


\section{System characterization - dynamic range}

The upper end of the streak camera dynamic range is determined by one of two limits. There is a hard linit to how intense a light pulse is obtainable from an MCP image intensifier when operated in a nulsed mode. This is because the MCP behaves as a segmented capacitor, charged to several hundred volts with a glass/vacuum dielectric. For excitation shorter than the recharge time (about 10 msec), the maximum charge that can be extracted from the MC.r is the charge stored in it. Phosphors used in streak tubes generally have decay times much shorter than $10 \mathrm{msec}$. The charge stored in the MCP is typically about $4 \times 10^{-9}$ coulombs $/ \mathrm{cm}^{2}$, or 50000 electrons/MCP channel. Therefore the maximum charge availabie from the $M C P$ is essentially independent of intensifier gain. This puts an upper limit on the number of photonsiunit area imaged onto the CCD or other recording media.

There is another limitation which may restrict the upper end of the dynamic range. This is a "soft" 1 imit caused by self-repulsion of the electron cloud in the streak camera ${ }^{3-6}$. When current density in the streak tube is large due to high instantaneous illumination of the photo cathode, the elecitrons will disperse both axially and radially. They spread over a larger area of the phosphor screen than they should. The resu?t is a measurement indicaing lower peak intensity and longer duration thes the actual input signals. The integral of the signal is, however, unchanged. This limitation is predominant for short light pulses and fast streak speeds. For long pulses and slower speeds, MCP saturation is more important. Streak camera users should be aware of which liqit applies in their application. The effects are quite different and will have different impact on signal recovery efforts.

There are also two factors which limit the lower end of streak camera dynamic range. The first is quantum noise, due to detection of single photo-electrons emitted from the streak camera photo-cathode. This depends on image intensifier gain and is dominant when the intensifier gain is high. The recording system noise forms the other lower limit. This is independent of intensifier gain and in the case of a CCD readout system is found by measuring the statistics of the electrical signal. The CCD camera we are now using has an RMS noise of 5 analog-to-digital converter (ADC) counts, corresponding to 50 hole-electron pairs or -100 incident photons per pixel. Since a background image must be subtracted during data reduction the effective noise is increased by $\sqrt{2}$ to 7 AOC counts.

We have found that the new CCD camera has sufficient dynamic range to both detect single streak camera photo-electrons (at high image intensifier gain) and to measure image intensifier saturation. At an image intensifier luminous gain of 15000 a single photo-electron produces about 2000 hole-electron pairs in the CCO. This results in a signal of about 200 analog-to-digital converter (ADC) counts, distributed over a maximum of 25 pixels. The ADC which digitizes the CCD signal has 14 -bit resolution for a maximum of 16383 counts per pi:iel. A totally discharged region of the MCP results in -10000 ADC counts per pixel, which is $60 \%$ of the full. range of the CCD camera. This results in a system dynamic range of about 1250, even without averaging. This permits us to characterize the dynamic range of a streak camera much more directly than in the past.

\section{Dynamic range measurtment technique}

A laser provides varying illumination to the streax camera by utilizing $70 \%$ etalon with 500 psec inter-pulse time. This provides a factor-of -2 reduction in the intensity of sequential input light pulses (see figure 1). Neutral density (ND) filters are used to provide a large input signal range. Typically six 100 -psec-long pulses (amplitude range of $32: 1$ ) are recorded on each streak record. By using No filters of 0,1 , and 2, the input signal covers a dynamic range of 3200 in three shots. Careful alignment of the etalon to prevent beam waiking across the slit is essential. It is also important to assure that the CCD columns are parallel to the streak camera sweep axis.

In order to obtain the system transfer function, peak intensity measurements are made at several values of image intensifier gain from 500 to 15000 . After correcting for sweep non-linearity and non-uniform light collection a relative system transfer function curve is obtained for each gain. Sweep linearity correction is necessary because signal amplitude varies inversely with sweep velocity. Non-uniform light collection is currently corrected by averaging the profiles of several shots that are well within the linear range of the system and applying a compensation to the measured amplitudes. Both of these steps have beeri done manually thus far. The rapid and accurate image digitization and computer access to the data will allow this to become more automated in the future.

The system noise, which is used to define the lower end of the dynamic range, is obtained by histograming a dark field. The result is a distribution which describes the noise of the system. For relatively low image intensifier gains, this is simply the noise of the CCD camera. As the intensifier gain is increased, single photo-electrons produce detectable CCO sinnal, raising the noise level. We are still evaluating how to best quantify this latter component of the noise. 

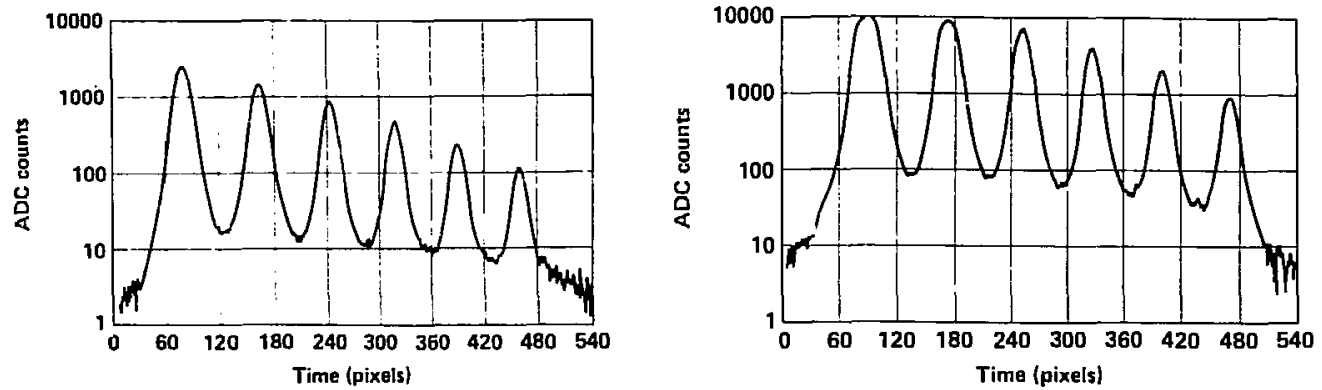

Fiqure 1 - A wide signal dynamic range is obtained by using a pulsed laser and a $70 \%$ etalon. Two cases show operation in the linear range (a) and when the image intensifier is saturated (b).

\section{Characterization of resolution}

We have not attempted to change the standard techniques for determining the temporal resolution of streak cameras ${ }^{7}$. We do, however, make measurements that allow us to report the modulation transfer functions (both axes) of the CCD camera-lens system, as well as along the slit at the input to the streak camera. This is done by installing an opaque edge either on the intensifier output or at a plane imaged onto the sTit (Figure 2). By differentiating the CCD signal along a column or row, then performing a Fourier transform, we obtain the MTF of the system being measured. A significant advantage of this approach is the ability to observe large scale effects such as lens flare, fiber optic cross talk, and electron scattering in the streak tube. These effects are typically masked when using resolution charts to measure resolution. They show up on a MTF curve as poor modulation at low spatial frequencies, below two lp/min at the streak camera input. This reduces the ability to accurately measure weak signals in the vicinity of intense ones. The limiting visual resolution of the system, generally defined as the spatial frequency where the MTF has a value of 0.04 , is also available from the MTF.

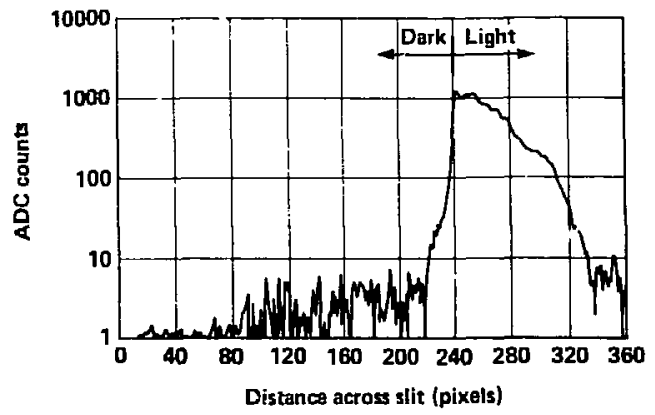

Figura 2 - The edge response (logarithmic vertical scale) of the streak camera system. The opaque edge is located at a slit which is imaged onto the photocathode. The image is produced by a $100-p 5 e c$ long light pulse and the camera is streaked at $15 \mathrm{~mm} / \mathrm{nsec}$. 
The data obtained from the characterization described above is presented to the streak camera user in the form of two plots (see figures 3 and 4 ). Figure 3 gives the system transfer function for iour image intensifier gain settings. The input intensity is in relative intensity, although in the future we plan to make this an absolute measurement. The output is in ADC counts per pixel. Image intensifier saturation is seen primarlily at high intensifier gains. As the intensifier gain decreases, the transfer function rolls off more gradually due to pulse broadening of the electron cloud in the streak tube.

The signal levels where $10 \%$ pulse compression (horizontal arrows) and 10\% pulse broadening (vertical arrows) occur are indtcated. Ten percent pulse compression will occur whenever the output of the intensifier exceeds $40 \%$ of MCP saturation. This agrees with one model of an MCP becoming non-linear 8 . The non-linearity may occur at a lower output level if it is due to electron cloud self-repulsion in the streak tube. Ten percent pulse broadening will occur at a fixed input intensity if it is due to self-repulsion of electrons in the streak tube. If it is due to MCP non-linearity it will occur at a lower intensity. The user shouid determine whether pulse compression or broadening is more detrimental in a particular application, and choose the intensifier gain accordingly.

The system noise level is also provided on this graph. The noise is due to the CCD camera except at high intensifier gains. At high gains the photo-electron quantum noise becomes significant. The diagonal line, shown here at the lower left, was determined by histograming faint images at high gains. We are not satisfied with this approach and plan to find a better one.

It is important to note that the noise is based on single pixel statistics. The user may reduce the effective system noise by averaging pixels, thus increasing dynamic range. Averaging over $n$ rows and $m$ columns will decrease the effective noise by $\sqrt{n \times m}$. The increased dynamic range is obtained at the expense of reduced resolution.

In addition to the system transfer function, several modulation transfer functions (see for example fiqure 4) are also provided to the user. These are obtained as described above, with some manipulation of the data to make the lower intensity side of the edge response dominate the computation, minimizing the effects of image non-uniformity.

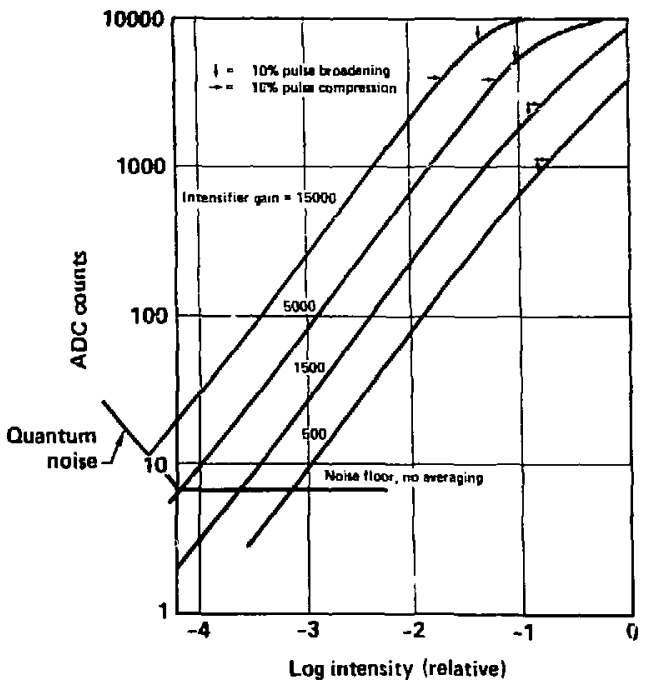

Figure 3 - The system transfer function provides information recuired to properly apply a streak camera and interpret data fro'n it.

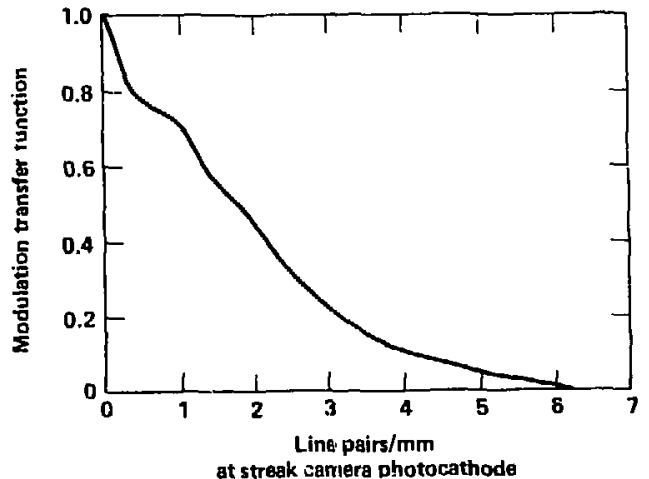

Figure 4 - The modulation transfer function calculated from the data of figure 2 . The dip in the vicinity of $0.5 \mathrm{lp} / \mathrm{mm}$ ts attributed to electron scattering from the MCP input face. 


\section{Sumary}

We are trying to present data to the streak camera user in a form that makes 1 t easier to properly set up the camera and interpret the data from it. The recent acquisition of an accurate, all electronic, computer interfaced camera has made this possible. Future work will include a better means to measure the effects of photo-electron noise.

\section{Acknowledgments}

The authors would like to thank John Severyn, Nick Selchow, Roger Griffith, Sue Bremer, and Don Browning for contributing to the CCD camera evaluation and measurements deseribed here. This work was performed under the auspices of the U.S. Department of Energ' by the Lawrence Livermore Laboratory under contract number W-7405-ENG-48.

\section{References}

1. Thomas, S.W., et al, "The LLL compact 10 psec strear camera - 1974 update," in Proc. 11th Int"l. Cong. on High Speed Photography, London, 1974, p. 101.

2. Jones, B.A., "A lens coupled streak camera readout system utilizing a thermoelectrically cooled CCD," Conf, on High Speed Photography, Videography and Photonics III, SPIE Proc. 569, August, 1985.

3. Bradley, D.J. et al, "Optimized electron-optical computations of image tube Stesigns for high temporal resolution chronoscopy," Proc. of the 13th Cong. on High Speed Photography and hotonics, 1978, p. 451.

4. Kalibjian, R., "Space-charge temporal broadening effects in streak-camera tube, " Proc. of the 13th Cong. on High Speed Photography and Photonics, 1978, pp. 452-455.

5. Degtyareva, V.P. and Platonov, V.N., "Computer evaluation of Coulomb repu"sion effects in picosecond streak image camera," Proc. 15th Int i). Cong. on High Speed Photography and Photonics, (1982) SP IE Vol. 348, pp. 1001-1004.

6. Kalibjian, R. and Peterson, G.G., "Analysis of space charge in photonic tubes," J. Appl. Phys. 54(8) Aug. 1983 , pp. $4295-4301$.

7. Fleurot, N., "Workshop on picosecond streak cameras," Proc. 16th Int'1. Cong. on High Speed Photography and Photonics, (7984) SPIE Vol. 497, pp. 374-375.

Attendees at this workshop concluded:

Temporal resolution should be specified by one of the two following criterla:

- F.W.H.M. of the pulse response to a delta intensity input function

- or by Rayleigh limit $\Delta T$ such that the contrast

$$
c=\frac{V m}{V p}=20 \%
$$

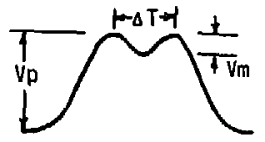

The following complementary narameters should be simultaneously specified:

- sample width in spatial direction

- sweep speed

- flux density level

- wavelength

- spatial resolution corresponding to this temooral resolution.

Information concerning degradation of temporal resolution off center should be given.

8. Csorba, I.P., "Current gain parameters oí microchannel plates," Applied Optics, Vol. 19, No. 22, Nov. 1980 , p. 3863. 